\title{
BioPhotonics Workstation: a university tech transfer challenge
}

\author{
Glückstad, Jesper; Bañas, Andrew Rafael; Tauro, Sandeep; Palima, Darwin
}

Published in:

Proceedings of SPIE - The International Society for Optical Engineering

Link to article, DOI:

$10.1117 / 12.898647$

Publication date:

2011

Document Version

Publisher's PDF, also known as Version of record

Link back to DTU Orbit

Citation (APA):

Glückstad, J., Bañas, A. R., Tauro, S., \& Palima, D. (2011). BioPhotonics Workstation: a university tech transfer challenge. Proceedings of SPIE - The International Society for Optical Engineering, 8097, 809714. https://doi.org/10.1117/12.898647

\section{General rights}

Copyright and moral rights for the publications made accessible in the public portal are retained by the authors and/or other copyright owners and it is a condition of accessing publications that users recognise and abide by the legal requirements associated with these rights.

- Users may download and print one copy of any publication from the public portal for the purpose of private study or research.

- You may not further distribute the material or use it for any profit-making activity or commercial gain

- You may freely distribute the URL identifying the publication in the public portal 


\title{
BioPhotonics Workstation: a university tech transfer challenge
}

\author{
Jesper Glückstad, Andrew Bañas, Sandeep Tauro, Darwin Palima \\ DTU Fotonik, Dept. of Photonics Engineering, \\ Programmable Phase Optics \\ DK-2800 Kgs. Lyngby, Denmark \\ jesper.gluckstad@fotonik.dtu.dk \\ www.ppo.dk
}

\begin{abstract}
Conventional optical trapping or tweezing is often limited in the achievable trapping range because of high numerical aperture and imaging requirements. To circumvent this, we are developing a next generation BioPhotonics Workstation platform that supports extension modules through a long working distance geometry. This geometry provides three dimensional and real time manipulation of a plurality of traps facilitating precise control and a rapid response in all sorts of optical manipulation undertakings. We present ongoing research and development activities for constructing a compact next generation BioPhotonics Workstation to be applied in three-dimensional studies on regulated microbial cell growth including their underlying physiological mechanisms, in vivo characterization of cell constituents and manufacturing of nanostructures and new materials.
\end{abstract}

Keywords: Spatial light modulator, optical manipulation, topology optimization

\section{INTRODUCTION}

The fascinating ability to manipulate microscopic structures in three dimensions using only light as "invisible hands" gives rise to new and until recently unachievable micro-biological applications [1,2]. Microscopic scaffoldings and tools [3] that can be reassembled with multiple traps can simulate biological microenvironments [4] and can potentially pave the way for advanced research in stem cell development and cancerous tissue on the cellular level. Using contemporary micro-fabrication processes elegantly provided by two-photon femto-second photo-polymerization acting as 3D scanning micro-printers, specially designed microscopic tools can be fabricated and subsequently driven around biological samples for probing or sending stimulus. Similar to freely movable hand tools, 3D controllable micro-tools can be used to trigger biological, chemical or mechanical reactions in a localized and controlled manner.

For a plurality of years, manipulation of microscopic particles has been carried out using laser traps created from a single strongly focused beam using a high numerical aperture objective. Such single-beam traps rely on light intensity gradients and are usually referred to as optical tweezers. The advent of computer addressable spatial light modulators has enabled means of creating a plurality of reconfigurable traps capable of translating simple bead like structures and rotating structures with multiple optical handles. Since standard optical tweezers are based on traps that rely on high intensity regions and high numerical aperture objective lenses, the range of motion in the axial direction is inherently very limited. We overcome this limitation using a counter-propagating beam geometry on our BioPhotonics Workstation [5].

Optical Trapping and Optical Micromanipulation VIII, edited by Kishan Dholakia, Gabriel C. Spalding, Proc. of SPIE Vol. 8097, 809714 - (c) 2011 SPIE · CCC code: 0277-786X/11/\$18 · doi: 10.1117/12.898647 


\section{MULTI-BEAM COUNTER-PROPAGATING BEAM TRAPPING}

The extended axial manipulation range on the BioPhotonics Workstation is obtained by using a low NA counterpropagating beam geometry. Counter-propagating (CP) traps take advantage of the long working distance wherein the light intensity does not deviate as much as compared to the high NA focusing. Trapped particles are translated axially by varying the relative intensities of the counter propagating beams (Fig 1). This axial degree of freedom enables more interactive manipulation flipping of planar microstructures and lifting puzzle pieces of reconfigurable microenvironments. Therefore, with the concerted transverse and axial manipulation provided by gradient forces and scattering forces respectively $3 \mathrm{D}$ optical manipulation is achieved while overcoming the short range limitations of high NA optical tweezer based trapping.

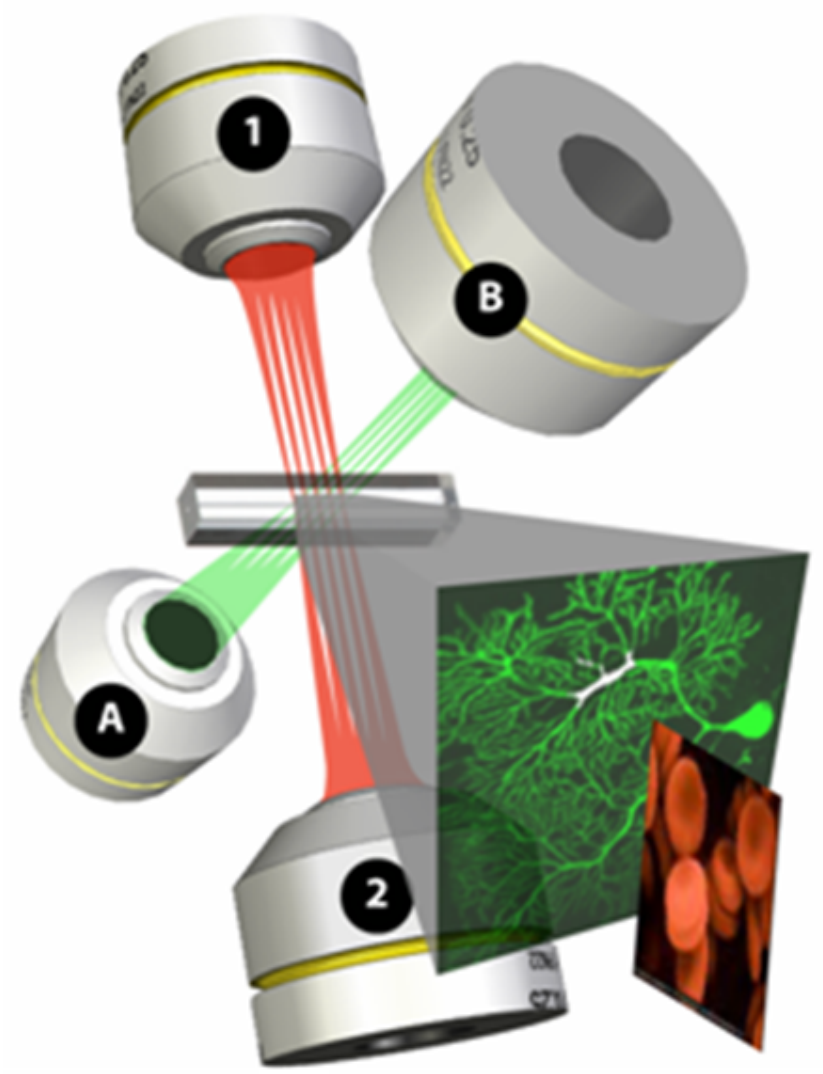

Figure 1: The counter-propagating beam traps on the BioPhotonics Workstation (provided by objectives 1 and 2) provides for a substantially extended axial trapping range compared to standard optical tweezers in addition to the possibility of using auxillary optics (objectives 3 and 4 ) in the side geometry for enhanced microscopic imaging modalities.

Long range 3D optical manipulation enables a host of novel applications, especially in microscopic biological studies. For example, microscopic scaffoldings fabricated with two-photon polymerization (2PP) can be reassembled with multiple traps, simulating biological microenvironments that can influence the behavior of cells (Fig 2). With micro or nano-fabrication processes such as $2 \mathrm{PP}$ or chemical micro-assembly, specially designed microscopic tools can be driven 
around biological samples for probing or sending stimulus. Similar to freely movable hand tools, 3D controllable microtools can be used to trigger biological, chemical or mechanical reactions in a localized and controlled manner.
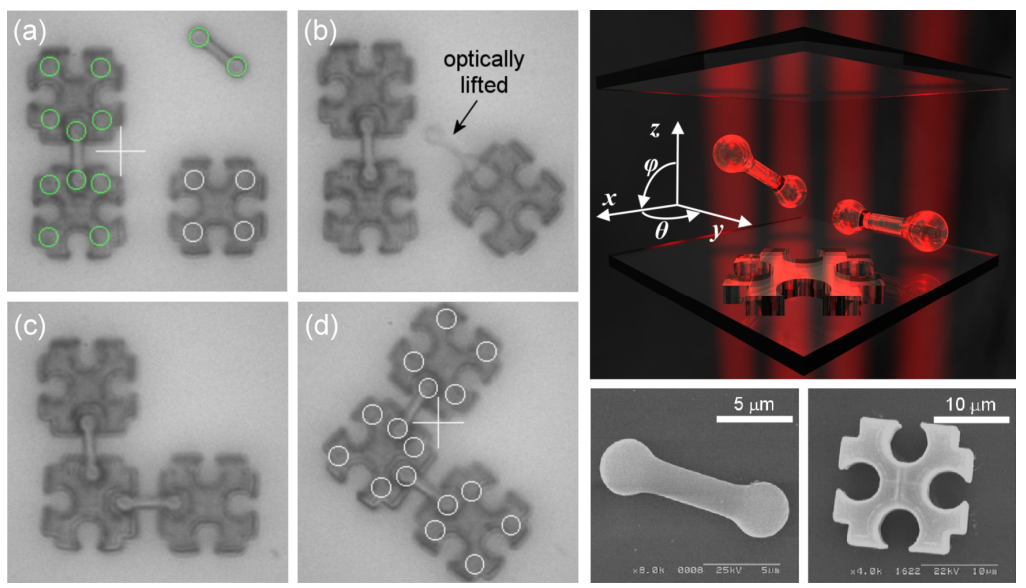

Figure 2: Image sequence from an experimentally recorded video illustrating optical microassembly of reconfigurable microenvironments fabricated using two-photon photo-polymerized components. The photos are taken from reference 4 and were featured by Nature Photonics later the same year.

\section{LAYOUT OF THE BIOPHOTONICS WORKSTATION}

A schematics of the BioPhotonics Workstation is illustrated in Fig. 3 and its configuration and optical modules has been dealt with extensively so we outline only the pertinent features here. Two independently addressable regions of a spatial beam modulator are optically mapped and relayed as a plurality of reconfigurable counter-propagating beams in the sample.

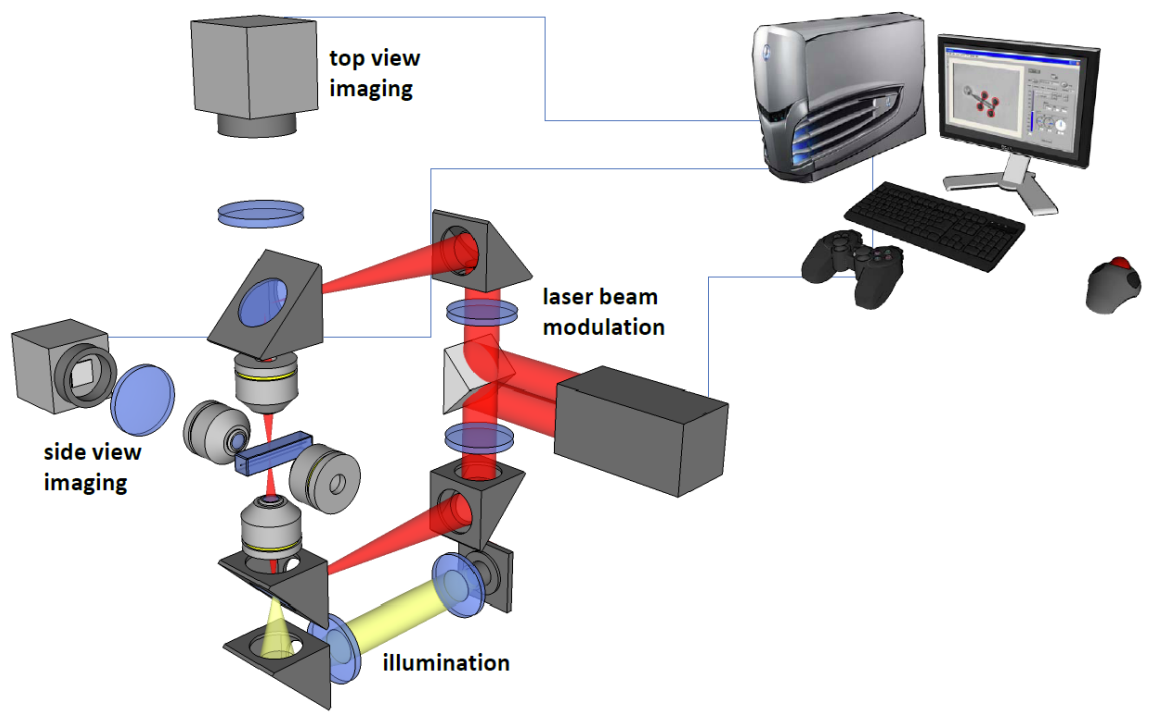

Figure 3: Schematics of the BioPhotonics workstation. The long working distance allows an extra microscope to be mounted perpendicular for side view or for an independent optical setup. The laser source is modulated and 
shaped by a spatial light modulator; the upper and lower parts of the beam are separated and projected into the sample from opposite directions. A periscopic design, with two mirrors in each arm, simplifies the optical alignment.

The scaling between the spatially light modulating pixels and the sample plane are specified by choosing appropriate focal lengths of the relaying lenses. The user can independently control the number, size, shape, intensity and spatial position of each CP-beamlet through a LabView interface. Each CP-beamlet can independently trap and manipulate a microscopic object, in parallel with the other trapping CP-beamlets. Computational overhead is minimized by using an imaging geometry allowing intensity patterns defining the optical traps to be directly mapped into an addressable light shaping module. Hence, real time reconfigurable counter-propagating beam traps. Another important advantage of using low NA objectives is the increased workspace that gives more freedom on the sample containers or other contraptions for performing other biological studies. For example, advanced micro-spectroscopic or multi-photon characterization methods [6] can be implemented independently in the side-geometry. Cells can be subjected to stress and then characterized for different health indicators while trapped laser whose effect is known or being studied [7].

Recently we demonstrated dynamic axial stabilization of counter-propagating beam traps by tracking the trajectory of particles trough the side view imaging [8]. Additional stabilization is necessary as we stretch the axial trapping reach because regions with less intense light are less stable in holding particles in place. Computer vision tracks axial positions of multiple particles for use in a feedback algorithm that adjusts the respective counter-propagating beam pair intensities as needed (Fig 4). This allows particles to be moved-to or held in user defined axial positions within the $250 \mu \mathrm{m}$ height of a micro-fluidic channel.

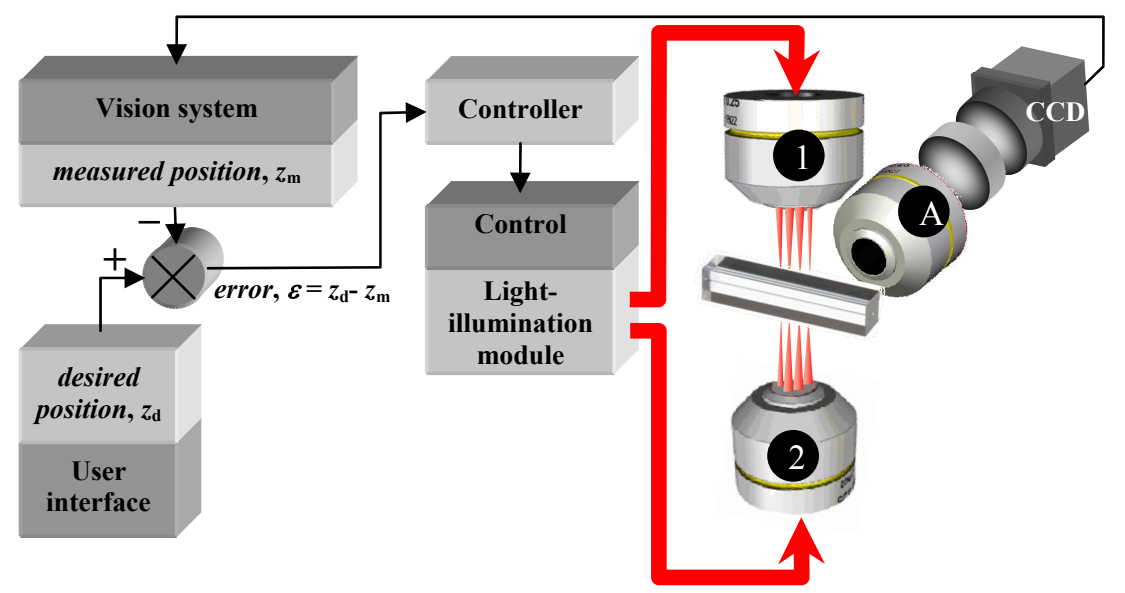

Figure 4. Active trap stabilization on the BioPhotonics Workstation using vision feedback [8]. An array of actively regulated traps are relayed through well-separated objectives ( 1 and 2 ) that provides ample space for sideview microscopy (objective lens A, zoom lens and CCD camera). Computer vision provides real-time position feedback for regulating the traps.

\section{MINITUARIZED BIOPHOTONICS WORKSTATION}

Up to this point, the BWS units in use are developed mostly with standard optical components and without requiring them to be compact or portable. The lack of such constraints allow high power optical traps, high quality imaging and an 
easily extensible hardware platform. User demands, however, are different and we may trade off some practical functionality that are not provided by a fully featured BioPhotonics Workstation. Developing a platform with a smaller footprint, for example, is a crucial step to make it more accessible for other intended users. This requires tweaking the BWS geometry and using more compact opto-mechanical components. Right now we are developing a miniature prototype BWS using standard and off-the-shelf components.

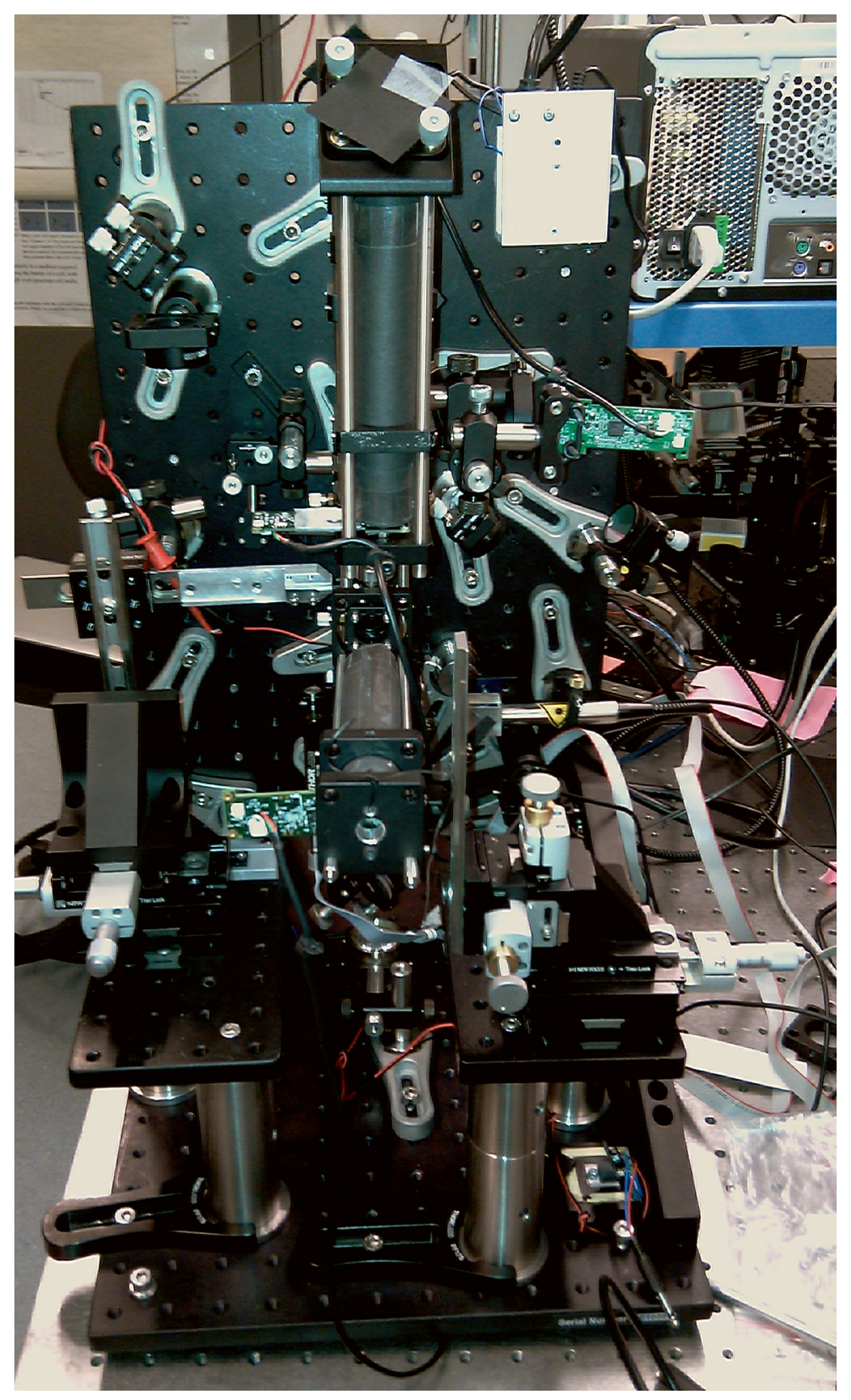

Figure 5. A miniaturised and cost-effective BioPhotonics Workstation. By using compact opto-mechanical components, and tweaking the trapping geometry, a smaller table-top BioPhotonics Workstation has been implemented. 
In principle, opto-mechanical components can be made as small as desired using customized manufacturing techniques. The challenge in miniaturization lies with the laser sources since its output power is correlated with its size. Hence, an important step for miniaturization is to efficiently use the trapping lasers, allowing smaller power lasers to be used. The conventional CP geometry requires the trapped particle to be dislocated from the foci of the opposing objectives. Such a region however, has less intense light, and hence less trapping stiffness. One way of dealing with this without increasing the input laser power is to use a dynamic software feedback approach as mentioned in the previous section [8]. Another approach is to identify alternate regimes of stable trapping that exploit the higher intensities as the particle moves toward opposite image planes $[9,10]$. The results suggest that further optimizations for stability can be done modifying the 3D light distribution of the counter-propagating traps. Another area of improvement in terms of efficiency is with the input light that goes into the spatial light modulator. Laser output typically has a Gaussian amplitude distribution and a circular area while pixel oriented spatial light modulators are rectangular. Hence, losses are introduced when the input laser is magnified to illuminate the whole SLM surface. GPC can efficiently convert a Gaussian amplitude distribution into a rectangle that matches the SLM [11-14].
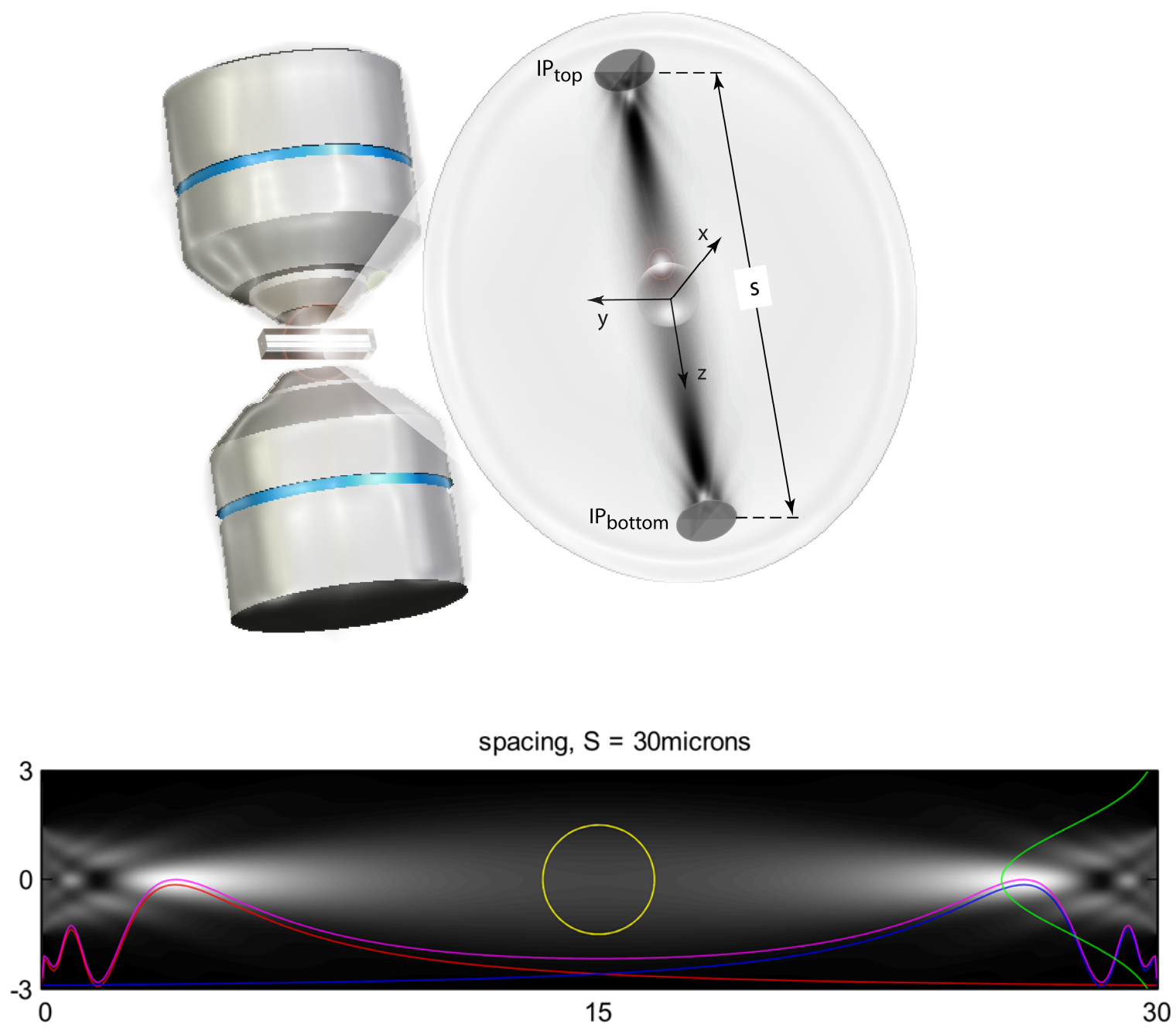
Figure 6. The analysis of the counter-propagating light distribution and forces reveals alternate regions for stable optical trapping near the image planes where light is more intense [9]. (Top) 3D view of counter-propagating discshaped beams projected through opposite microscope objectives onto image planes, "IP top" and "IP bottom", separated by distance, $S$, to create a stable optical trap between the image planes. (Bottom) An axial slice through the simulated volume intensity between two 3micron diameter light discs; overlays show the expected stable trapping position for a microsphere, together with plots of intensity linescans (red: axial intensity of right-directed beam; blue: axial intensity of the left-directed beam; magenta: total axial intensity; green: transverse intensity linescan halfway between the discs)

\section{TOPOLOGY OPTIMIZATION ON THE BIOPHOTONICS WORKSTATION}

Recently, we also started to undertake studies on exciting new avenue for three-dimensional light-matter engineering on the BioPhotonics Workstation using computational tricks from so-called topology optimization. In short, this is a computational method using sets of iterated differential equations to gradually determine the optimal topology for a given engineering task or design. Topology optimization has already been successfully applied in a plurality of engineering disciplines by e.g. optimizing the distribution of material for a wide span of situations from the load-bearing structure of an airplane wing and down to the design of tiny micro-robotic grippers. The method is usually based on repeated computer calculations and the results often lead to surprisingly new designs of known and existing structures. Recently, a substantial amount of work has been focused on extending topology optimization to applications involving fluid flows and wave-propagation but has apparently not yet been applied to optimizing sculpted light-matter interaction. A first step for this undertaking would be to define the external boundaries of the design one has in mind. This should then be followed by fully 3D computer-based optimization used to improve the initial design in a step-wise manner. After a plurality of iterations the process should gradually arrive at the optimal way to shape the object given a particular light-matter interaction. We anticipate that this will make us see scientists and nano-engineers designing and sculpting a next generation of top-tuned light-driven micro-machines using these emerging 3D computational and engineering schemes [15].

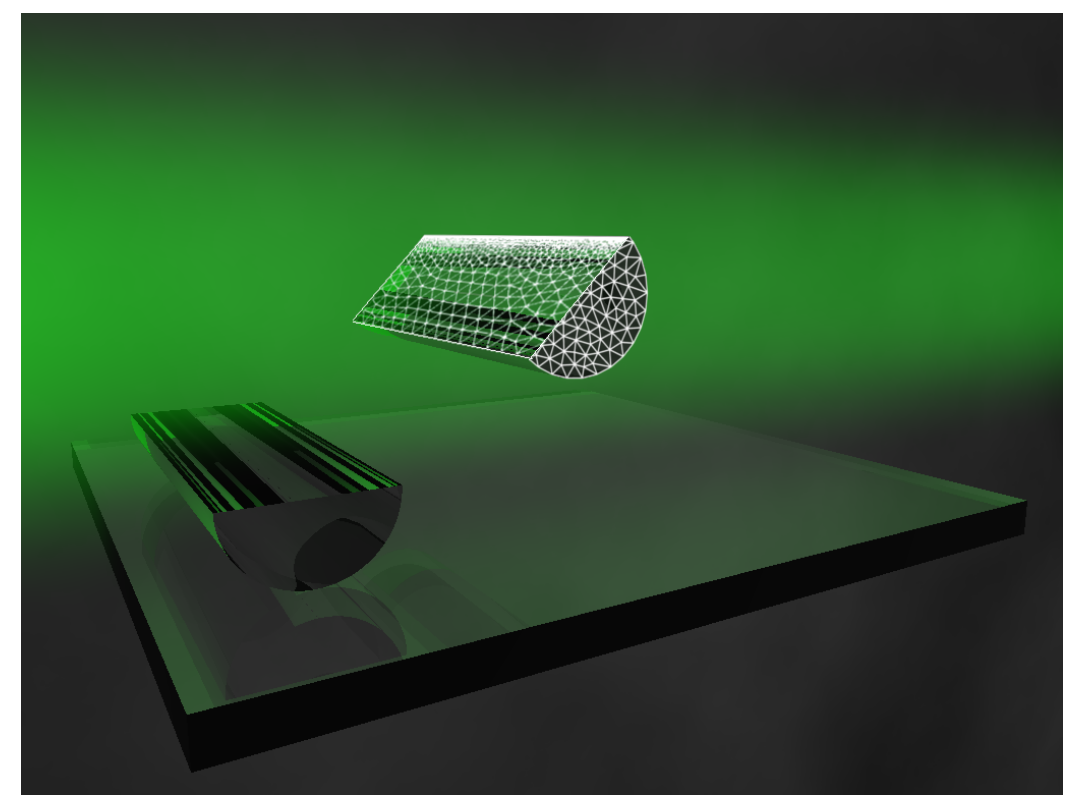

Figure 7: The conceptualized use of topology optimization for optimizing light matter interaction as envisioned in ref.

15. 


\section{CONCLUSIONS}

Our recently developed and minituarized BioPhotonics Workstation enables long range lateral and axial optical manipulation and extensible functionality through its side view imaging objectives. This is achieved by using a counterpropagating geometry coupled with long working distance optics. Direct mapping of the trap intensities to the sample allows real time manipulation of a plurality of independent traps, facilitating precise control and a rapid response in optical manipulation. Using extensions through the side view imaging, biological applications requiring different imaging requirements can be performed. Side view information has also been used to develop a stabilization scheme based on tracking the trapped particles' motions. Ongoing research aims at developing a Next Generation BioPhotonics Workstation that is even more compact and efficient in using laser power.

\section{REFERENCES}

1. A. Ashkin, "Acceleration and trapping of particles by radiation pressure," Phys. Rev. Lett. 24, 4,156-159 (1970).

2. J. Glückstad, "Sorting particles with light", Nature Materials 3, 9-10 (2004).

3. P. Rodrigo, L. Gammelgaard, P. Bøggild, I. P.-Nielsen, and J. Glückstad, "Actuation of microfabricated tools using multiple GPC-based counterpropagating-beam traps," Opt. Express 13, 6899-6904 (2005).

4. P. Rodrigo, L. Kelemen, D. Palima, C. Alonzo, P. Ormos, and J. Glückstad, "Optical microassembly platform for constructing reconfigurable microenvironments for biomedical studies," Opt. Express 17, 6578-6583 (2009).

5. J. Glückstad, P. Rodrigo, I. P.-Nielsen, "Optical 3D manipulation and observation in real-time (Invited)", $J$. Robotics Mechatronics 18 (no.6), 692-697 (2006).

6. E. Papagiakoumou, F. Anselmi, A. Begue, V. de Sars, J. Glückstad, E. Isacoff, V. Emiliani, Nature Methods 7, 848-854 (2010).

7. T. Aabo, I. P.-Nielsen, J. Dam, D. Palima, H. Siegumfeldt, J. Glückstad and N. Arneborg, "Effect of long- and short-term exposure to laser light at $1070 \mathrm{~nm}$ on growth of Saccharomyces Cerevisiae", J. Biomed. Opt. 15, 041505 (2010).

8. S. Tauro, A. Bañas, D. Palima and J. Glückstad, "Dynamic axial stabilization of counter-propagating beamtraps with feedback control," Opt. Express 18, 18217-18222 (2010).

9. D. Palima, T.B. Lindballe, M.V. Kristensen, S. Tauro, A. Banas, H. Stapelfeldt, S. R. Keiding, and J. Gluckstad, "Counter-propagating patterns in the BioPhotonics Workstation: getting more out of light for trapping and manipulation", Proc. SPIE 7762, $77620 \mathrm{U}$ (2010).

10. D. Palima, T.B. Lindballe, M.V. Kristensen, S. Tauro, H. Stapelfeldt, S.R. Keiding and J. Glückstad, "Alternative modes for optical trapping and manipulation using counter-propagating shaped beams," Journal of Optics, vol. 13, issue 4, (2011)

11. D. Palima and J. Glückstad, "Gaussian to uniform intensity shaper based on generalized phase contrast," Opt. Express 16, 1507-1516 (2008).

12. J. Glückstad and P. C. Mogensen, "Reconfigurable ternary-phase array illuminator based on the generalised phase contrast method", Opt. Comm. 173, 169-175 (2000).

13. J. Glückstad, "Adaptive array illumination and structures light generated by spatial zero-order self-phase modulation in a Kerr medium", Opt. Comm. 120, 194-203 (1995).

14. H. Ulriksen, J. Thøgersen, S. Keiding, I. P.-Nielsen, J. Dam, D. Palima, H. Stapelfeldt, J. Glückstad, J. Europ. Opt. Soc. Rap. Public. 08034 Vol 3 (2008).

15. J.Glückstad, "Optical manipulation: Sculpting the object”, Nature Photonics 5, 7-8 (2011). 